\title{
GENERALIZED ANALYTIC FOURIER-FEYNMAN TRANSFORMS AND CONVOLUTIONS ON A FRESNEL TYPE CLASS
}

\author{
Seung Jun Chang and Il Yong Lee
}

\begin{abstract}
In this paper, we define an $L_{p}$ analytic generalized FourierFeynman transform and a convolution product of functionals in a $\mathrm{Ba}-$ nach algebra $\mathcal{F}\left(C_{a, b}[0, T]\right)$ which is called the Fresnel type class, and in more general class $\mathcal{F}_{A_{1}, A_{2}}$ of functionals defined on general function space $C_{a, b}[0, T]$ rather than on classical Wiener space. Also we obtain some relationships between the $L_{p}$ analytic generalized Fourier-Feynman transform and convolution product for functionals in $\mathcal{F}\left(C_{a, b}[0, T]\right)$ and in $\mathcal{F}_{A_{1}, A_{2}}$.
\end{abstract}

\section{Introduction}

Let $C_{0}[0, T]$ denote one-parameter Wiener space; that is the space of $\mathbb{R}$ valued continuous functions $x(t)$ on $[0, T]$ with $x(0)=0$. The concept of an $L_{1}$ analytic Fourier-Feynman transform (FFT) for functionals on Wiener space was introduced by Brue in [2]. Further work involving the $L_{2}-L_{2}$ theory and the $L_{p}-L_{p^{\prime}}$ theory, $1 / p+1 / p^{\prime}=1$, includes [3, 16]. In [13], Huffman, Park and Skoug defined a convolution product $(\mathrm{CP})$ for functionals on Wiener space, and they obtained various results for the FFT and CP [13, 14, 15]. On the other hand, in [1], Ahn investigated the $L_{1}$ FFT theory on the Fresnel class $\mathcal{F}(B)$ of an abstract Wiener space, and in [11] Chang, Song and Yoo studied the FFT and the first variation on an abstract Wiener space and corresponding Fresnel class $\mathcal{F}(B)$. There has been a tremendous amount of papers in the literature on the FFT and CP theory on classical and abstract Wiener spaces. Furthermore, in [18], Kallianpur and Bromley introduced a larger class $\mathcal{F}_{A_{1}, A_{2}}$ than the Fresnel class $\mathcal{F}(B)$ for a successful treatment of certain physical problems by means of a Feynman integral.

In recent paper [8], Chang and Skoug established various results involving generalized analytic Feynman integrals and generalized analytic FFTs(GFFT)

Received March 17, 2007; Revised January 26, 2011.

2010 Mathematics Subject Classification. Primary 60J25, 28C20.

Key words and phrases. generalized Brownian motion process, generalized analytic Feynman integral, generalized analytic Fourier-Feynman transform, convolution product, Fresnel type class. 
for functionals defined on a very general function space $C_{a, b}[0, T]$ rather than on the Wiener space $C_{0}[0, T]$. The function space $C_{a, b}[0, T]$ was introduced by Chang and Chung in [6]. In [4], Chang and Choi studied a multiple $L_{p}$ analytic GFFT on the Banach algebra $\mathcal{S}\left(L_{a, b}^{2}[0, T]\right)$ which was introduced in [8]. On the other hand, in $[9,10]$, Chang and Lee defined a Fresnel type class $\mathcal{F}\left(C_{a, b}[0, T]\right)$ of functionals defined on $C_{a, b}[0, T]$ and studied GFFT, conditional GFFT, and multiple $L_{p}$ analytic GFFT on the Fresnel type class $\mathcal{F}\left(C_{a, b}[0, T]\right)$.

In this paper, we define an $L_{p}$ analytic GFFT and a $\mathrm{CP}$ of functionals defined on a product function space $C_{a, b}^{2}[0, T] \equiv C_{a, b}[0, T] \times C_{a, b}[0, T]$ and establish various relationships between the GFFT and CP of functionals in $\mathcal{F}_{A_{1}, A_{2}}$ which is a class of functionals defined on the function space $C_{a, b}^{2}[0, T]$. The Wiener process used in $[3,13-18,1,4$, and 5] is stationary in time and is free of drift, while the stochastic process used in [6-11], and in this paper, is nonstationary in time and is subject to the drift $a(t)$. Of course, if $a(t) \equiv 0$ and $b(t)=t$ on $[0, T]$, the $C_{a, b}[0, T]$ reduces to Wiener space $C_{0}[0, T]$.

\section{Definitions and preliminaries}

Let $D=[0, T]$ and let $(\Omega, \mathcal{B}, P)$ be a probability measure space. A realvalued stochastic process $Y$ on $(\Omega, \mathcal{B}, P)$ and $D$ is called a generalized Brownian motion process if $Y(0, \omega)=0$ almost everywhere and for $0=t_{0}<t_{1}<\cdots<$ $t_{n} \leq T$, the $n$-dimensional random vector $\left(Y\left(t_{1}, \omega\right), \ldots, Y\left(t_{n}, \omega\right)\right)$ is normally distributed with the density function

$$
\begin{aligned}
K(\vec{t}, \vec{\eta})= & \left((2 \pi)^{n} \prod_{j=1}^{n}\left(b\left(t_{j}\right)-b\left(t_{j-1}\right)\right)\right)^{-1 / 2} \\
& \cdot \exp \left\{-\frac{1}{2} \sum_{j=1}^{n} \frac{\left(\left(\eta_{j}-a\left(t_{j}\right)\right)-\left(\eta_{j-1}-a\left(t_{j-1}\right)\right)\right)^{2}}{b\left(t_{j}\right)-b\left(t_{j-1}\right)}\right\}
\end{aligned}
$$

where $\vec{\eta}=\left(\eta_{1}, \ldots, \eta_{n}\right), \eta_{0}=0, \vec{t}=\left(t_{1}, \ldots, t_{n}\right), a(t)$ is an absolutely continuous real-valued function on $[0, T]$ with $a(0)=0, a^{\prime}(t) \in L^{2}[0, T]$, and $b(t)$ is a strictly increasing, continuously differentiable real-valued function with $b(0)=$ 0 and $b^{\prime}(t)>0$ for each $t \in[0, T]$.

As explained in [21, pp. 18-20], $Y$ induces a probability measure $\mu$ on the measurable space $\left(\mathbb{R}^{D}, \mathcal{B}^{D}\right)$ where $\mathbb{R}^{D}$ is the space of all real-valued functions $x(t), \quad t \in D$, and $\mathcal{B}^{D}$ is the smallest $\sigma$-algebra of subsets of $\mathbb{R}^{D}$ with respect to which all the coordinate evaluation maps $e_{t}(x)=x(t)$ defined on $\mathbb{R}^{D}$ are measurable. The triple $\left(\mathbb{R}^{D}, \mathcal{B}^{D}, \mu\right)$ is a probability measure space. This measure space is called the function space induced by the generalized Brownian motion process $Y$ determined by $a(\cdot)$ and $b(\cdot)$.

We note that the generalized Brownian motion process $Y$ determined by $a(\cdot)$ and $b(\cdot)$ is a Gaussian process with mean function $a(t)$ and covariance function $r(s, t)=\min \{b(s), b(t)\}$. By Theorem 14.2 [21, p. 187], the probability measure $\mu$ induced by $Y$, taking a separable version, is supported by $C_{a, b}[0, T]$ (which is 
equivalent to the Banach space of continuous functions $x$ on $[0, T]$ with $x(0)=0$ under the sup norm). Hence $\left(C_{a, b}[0, T], \mathcal{B}\left(C_{a, b}[0, T]\right), \mu\right)$ is the function space induced by $Y$ where $\mathcal{B}\left(C_{a, b}[0, T]\right)$ is the Borel $\sigma$-algebra of $C_{a, b}[0, T]$.

Given two $\mathbb{C}$-valued measurable functions $F$ and $G$ on $C_{a, b}[0, T], F$ is said to be equal to $G$ scale almost everywhere(s-a.e.) if for each $\rho>0, \mu(\{x \in$ $\left.\left.C_{a, b}[0, T]: F(\rho x) \neq G(\rho x)\right\}\right)=0[12,17]$. We write that $F \approx G$ if $F=G$ s-a.e..

Let $L_{a, b}^{2}[0, T]$ be the set of functions on $[0, T]$ which are Lebesgue measurable and square integrable with respect to the Lebesgue-Stieltjes measures on $[0, T]$ induced by $a(\cdot)$ and $b(\cdot)$; i.e.,

$$
L_{a, b}^{2}[0, T]=\left\{v: \int_{0}^{T} v^{2}(s) d b(s)<\infty \text { and } \int_{0}^{T} v^{2}(s) d|a|(s)<\infty\right\},
$$

where $|a|(t)$ denotes the total variation of the function $a(\cdot)$ on the interval $[0, t]$.

For $u, v \in L_{a, b}^{2}[0, T]$, let

$$
(u, v)_{a, b}=\int_{0}^{T} u(t) v(t) d[b(t)+|a|(t)] .
$$

Then $(\cdot, \cdot)_{a, b}$ is an inner product on $L_{a, b}^{2}[0, T]$ and $\|u\|_{a, b}=\sqrt{(u, u)_{a, b}}$ is a norm on $L_{a, b}^{2}[0, T]$. In particular, note that $\|u\|_{a, b}=0$ if and only if $u(t)=0$ a.e. on $[0, T]$. Furthermore, $\left(L_{a, b}^{2}[0, T],\|\cdot\|_{a, b}\right)$ is a separable Hilbert space.

Let $\left\{\phi_{j}\right\}_{j=1}^{\infty}$ be a complete orthogonal set of real-valued functions of bounded variation on $[0, T]$ such that

$$
\left(\phi_{j}, \phi_{k}\right)_{a, b}= \begin{cases}0 & , j \neq k \\ 1 & , j=k\end{cases}
$$

Then for each $v \in L_{a, b}^{2}[0, T]$, the Paley-Wiener-Zygmund (PWZ) stochastic integral $\langle v, x\rangle$ is defined by the formula

$$
\langle v, x\rangle=\lim _{n \rightarrow \infty} \int_{0}^{T} \sum_{j=1}^{n}\left(v, \phi_{j}\right)_{a, b} \phi_{j}(t) d x(t)
$$

for all $x \in C_{a, b}[0, T]$ for which the limit exists.

Remark 2.1. For each $v \in L_{a, b}^{2}[0, T]$, the PWZ stochastic integral $\langle v, x\rangle$ exists for $\mu$-a.e. $x \in C_{a, b}[0, T]$ and $\langle v, x\rangle$ is a Gaussian random variable on $C_{a, b}[0, T]$ with mean $\int_{0}^{T} v(s) d a(s)$ and variance $\int_{0}^{T} v^{2}(s) d b(s)$. Note that for all $u, v \in$ $L_{a, b}^{2}[0, T]$,

$$
\begin{aligned}
& \int_{C_{a, b}[0, T]}\langle u, x\rangle\langle v, x\rangle d \mu(x) \\
= & \int_{0}^{T} u(s) v(s) d b(s)+\int_{0}^{T} u(s) d a(s) \int_{0}^{T} v(s) d a(s) .
\end{aligned}
$$


Hence we see that for all $u, v \in L_{a, b}^{2}[0, T], \int_{0}^{T} u(s) v(s) d b(s)=0$ if and only if $\langle u, x\rangle$ and $\langle v, x\rangle$ are independent random variables.

Now, we state the definition of the generalized analytic Feynman integral.

Definition 2.2. Let $\mathbb{C}$ denote the complex numbers, let $\mathbb{C}_{+}=\{\lambda \in \mathbb{C}$ : $\operatorname{Re}(\lambda)>0\}$ and let $\tilde{\mathbb{C}}_{+}=\{\lambda \in \mathbb{C}: \lambda \neq 0$ and $\operatorname{Re}(\lambda) \geq 0\}$. Let $F: C_{a, b}[0, T] \rightarrow$ $\mathbb{C}$ be a measurable functional such that for each $\lambda>0$, the function space integral

$$
J(\lambda)=\int_{C_{a, b}[0, T]} F\left(\lambda^{-1 / 2} x\right) d \mu(x)
$$

exists. If there exists a function $J^{*}(\lambda)$ analytic in $\mathbb{C}_{+}$such that $J^{*}(\lambda)=J(\lambda)$ for all $\lambda>0$, then $J^{*}(\lambda)$ is defined to be the analytic function space integral of $F$ over $C_{a, b}[0, T]$ with parameter $\lambda$, and for $\lambda \in \mathbb{C}_{+}$we write

$$
E^{\mathrm{an}_{\lambda}}[F] \equiv E_{x}^{\mathrm{an}_{\lambda}}[F(x)]=J^{*}(\lambda) .
$$

Let $q \neq 0$ be a real number and let $F$ be a functional such that $E^{\operatorname{an}_{\lambda}}[F]$ exists for all $\lambda \in \mathbb{C}_{+}$. If the following limit exists, we call it the generalized analytic Feynman integral of $F$ with parameter $q$ and we write

$$
E^{\operatorname{anf}_{q}}[F] \equiv E_{x}^{\operatorname{anf}_{q}}[F(x)]=\lim _{\lambda \rightarrow-i q} E^{\operatorname{an}_{\lambda}}[F],
$$

where $\lambda \rightarrow-i q$ through values in $\mathbb{C}_{+}$.

Next, see $[8,9]$, we state the definition of the GFFT.

Definition 2.3. Let $q \in \mathbb{R}-\{0\}$. For $\lambda \in \mathbb{C}_{+}$and $y \in C_{a, b}[0, T]$, let

$$
T_{\lambda}(F)(y)=E_{x}^{\mathrm{an}_{\lambda}}[F(y+x)] .
$$

For $p \in(1,2]$, we define the $L_{p}$ analytic $\operatorname{GFFT}, T_{q}^{(p)}(F)$ of $F$, by the formula $\left(\lambda \in \mathbb{C}_{+}\right)$

$$
T_{q}^{(p)}(F)(y)=\text { l.i.m. }{ }_{\lambda \rightarrow-i q} T_{\lambda}(F)(y)
$$

if it exists; i.e., for each $\rho>0$,

$$
\lim _{\lambda \rightarrow-i q} \int_{C_{a, b}[0, T]}\left|T_{\lambda}(F)(\rho y)-T_{q}^{(p)}(F)(\rho y)\right|^{p^{\prime}} d \mu(y)=0,
$$

where $1 / p+1 / p^{\prime}=1$. We define the $L_{1}$ analytic $\operatorname{GFFT}, T_{q}^{(1)}(F)$ of $F$, by the formula $\left(\lambda \in \mathbb{C}_{+}\right)$

$$
T_{q}^{(1)}(F)(y)=\lim _{\lambda \rightarrow-i q} T_{\lambda}(F)(y)
$$

if it exists.

We note that for $1 \leq p \leq 2, T_{q}^{(p)}(F)$ is defined only s-a.e.. We also note that if $T_{q}^{(p)}(F)$ exists and if $F \approx G$, then $T_{q}^{(p)}(G)$ exists and $T_{q}^{(p)}(G) \approx T_{q}^{(p)}(F)$.

Next we give the definition of the $\mathrm{CP}$ on $C_{a, b}[0, T]$. 
Definition 2.4. Let $F$ and $G$ be measurable functionals on $C_{a, b}[0, T]$. For $\lambda \in \tilde{\mathbb{C}}_{+}$, we define their $\mathrm{CP}(F * G)_{\lambda}$ (if it exists) by

$$
(F * G)_{\lambda}(y)= \begin{cases}E_{x}^{\operatorname{an}_{\lambda}}\left[F\left(\frac{y+x}{\sqrt{2}}\right) G\left(\frac{y-x}{\sqrt{2}}\right)\right], & \lambda \in \mathbb{C}_{+} \\ E_{x}^{\operatorname{anf}_{q}}\left[F\left(\frac{y+x}{\sqrt{2}}\right) G\left(\frac{y-x}{\sqrt{2}}\right)\right], & \lambda=-i q, q \in \mathbb{R}, q \neq 0 .\end{cases}
$$

Remark 2.5. (i) When $\lambda=-i q$, we denote $(F * G)_{\lambda}$ by $(F * G)_{q}$.

(ii) Our definition of the $\mathrm{CP}$ is different than the definition given by Yeh in [20] and used by Yoo in [22]. In [20] and [22], Yeh and Yoo studied relationships between their CP and Fourier-Wiener transform.

The following generalized analytic Feynman integral formula is used several times in this paper.

$$
E_{x}\left[\exp \left\{i \lambda^{-1 / 2}\langle v, x\rangle\right\}\right]=\exp \left\{-\frac{\left(v^{2}, b^{\prime}\right)}{2 \lambda}+i \lambda^{-1 / 2}\left(v, a^{\prime}\right)\right\}
$$

for all $\lambda \in \tilde{\mathbb{C}}_{+}$and $v \in L_{a, b}^{2}[0, T]$ where

$$
\left(v, a^{\prime}\right)=\int_{0}^{T} v(s) a^{\prime}(s) d s=\int_{0}^{T} v(s) d a(s)
$$

and

$$
\left(v^{2}, b^{\prime}\right)=\int_{0}^{T} v^{2}(s) b^{\prime}(s) d s=\int_{0}^{T} v^{2}(s) d b(s) .
$$

In this paper, for each $\lambda \in \widetilde{\mathbb{C}}_{+}, \lambda^{-\frac{1}{2}}$ or $\lambda^{\frac{1}{2}}$ is chosen to have nonnegative real part.

\section{Transforms and convolutions of functionals in a Banach algebra}

In this section we introduce a Banach algebra $\mathcal{F}\left(C_{a, b}[0, T]\right)$ and evaluate the GFFT and CP of functionals belonging to the Banach algebra $\mathcal{F}\left(C_{a, b}[0, T]\right)$. We then obtain several relationships of the GFFT and CP. First, we give the definition of a Banach algebra $\mathcal{F}\left(C_{a, b}[0, T]\right)$ which is called the Fresnel type class on $C_{a, b}[0, T]$.

Let

$$
C_{a, b}^{\prime}[0, T]=\left\{w \in C_{a, b}[0, T]: w(t)=\int_{0}^{t} z(s) d b(s) \text { for some } z \in L_{a, b}^{2}[0, T]\right\} .
$$

For $w \in C_{a, b}^{\prime}[0, T]$, with $w(t)=\int_{0}^{t} z(s) d b(s)$ for $t \in[0, T]$, let $D_{t}: C_{a, b}^{\prime}[0, T] \rightarrow$ $L_{a, b}^{2}[0, T]$ be defined by the formula

$$
D_{t} w=z(t)=\frac{w^{\prime}(t)}{b^{\prime}(t)} .
$$


Then $C_{a, b}^{\prime} \equiv C_{a, b}^{\prime}[0, T]$ with inner product

$$
\left(w_{1}, w_{2}\right)_{C_{a, b}^{\prime}}=\int_{0}^{T} D_{t} w_{1} D_{t} w_{2} d b(t)
$$

is a separable Hilbert space. Furthermore, $\left(C_{a, b}^{\prime}[0, T], C_{a, b}[0, T], \mu\right)$ is an abstract Wiener space. For more details, see [19].

Note that for all $w, w_{1}, w_{2} \in C_{a, b}^{\prime}[0, T]$,

$$
\begin{gathered}
\left(\left(D_{t} w\right)^{2}, b^{\prime}\right)=\int_{0}^{T}\left(D_{t} w\right)^{2} d b(t)=\|w\|_{C_{a, b}^{\prime}}^{2}, \\
\left(D_{t} w, a^{\prime}\right)=\int_{0}^{T} D_{t} w d a(t)=\int_{0}^{T} D_{t} w D_{t} a d b(t)=(w, a)_{C_{a, b}^{\prime}}
\end{gathered}
$$

and

$$
\left\langle D_{t} w_{1}, w_{2}\right\rangle=\int_{0}^{T} D_{t} w_{1} d w_{2}(t)=\int_{0}^{T} D_{t} w_{1} D_{t} w_{2} d b(t)=\left(w_{1}, w_{2}\right)_{C_{a, b}^{\prime}} .
$$

Next, we define a class of functionals on $C_{a, b}[0, T]$ like a Fresnel class of an abstract Wiener space. Note that the linear operator given by the equation $(3.2)$ is an isomorphism. In fact, the inverse operator $D_{t}^{-1}: L_{a, b}^{2}[0, T] \rightarrow$ $C_{a, b}^{\prime}[0, T]$ is given by the formula

$$
D_{t}^{-1} z=\int_{0}^{t} z(s) d b(s)
$$

and $D_{t}^{-1}$ is a bounded operator since

$$
\begin{aligned}
\left\|D_{t}^{-1} z\right\|_{C_{a, b}^{\prime}} & =\left\|\int_{0}^{t} z(s) d b(s)\right\|_{C_{a, b}^{\prime}}=\left(\int_{0}^{T} z^{2}(t) d b(t)\right)^{\frac{1}{2}} \\
& \leq\left(\int_{0}^{T} z^{2}(t) d[b(t)+|a|(t)]\right)^{\frac{1}{2}}=\|z\|_{a, b} .
\end{aligned}
$$

Thus by the open mapping theorem, $D_{t}$ is also bounded and there exist positive real numbers $\alpha$ and $\beta$ such that $\alpha\|w\|_{C_{a, b}^{\prime}} \leq\left\|D_{t} w\right\|_{a, b} \leq \beta\|w\|_{C_{a, b}^{\prime}}$ for all $w \in C_{a, b}^{\prime}[0, T]$. Hence we see that the Borel $\sigma$-algebra on $\left(C_{a, b}^{\prime}[0, T],\|\cdot\|_{C_{a, b}^{\prime}}\right)$ is given by

$$
\mathcal{B}\left(C_{a, b}^{\prime}[0, T]\right)=\left\{D_{t}^{-1}(E): E \in \mathcal{B}\left(L_{a, b}^{2}[0, T]\right)\right\}
$$

and that for any complex Borel measure $\sigma$ on $L_{a, b}^{2}[0, T], \sigma \circ D_{t}$ is a complex Borel measure $\sigma$ on $C_{a, b}^{\prime}[0, T]$ and for any complex Borel measure $f$ on $C_{a, b}^{\prime}[0, T]$, $f \circ D_{t}^{-1}$ is a complex Borel measure $\sigma$ on $L_{a, b}^{2}[0, T]$.

Definition 3.1. Let $\mathcal{M}\left(C_{a, b}^{\prime}[0, T]\right)$ be the space of complex-valued, countably additive (and hence finite) Boreal measures on $C_{a, b}^{\prime}[0, T]$. The Banach algebra 
$\mathcal{F}\left(C_{a, b}[0, T]\right)$ consists of those functionals $F$ on $C_{a, b}[0, T]$ expressible in the form

$$
F(x)=\int_{C_{a, b}^{\prime}[0, T]} \exp \left\{i\left\langle D_{t} w, x\right\rangle\right\} d f(w)
$$

for s-a.e. $x \in C_{a, b}[0, T]$, where the associated measure $f$ is an element $\mathcal{M}\left(C_{a, b}^{\prime}[0\right.$, $T])$. We call $\mathcal{F}\left(C_{a, b}[0, T]\right)$ the Fresnel type class of the function space $C_{a, b}[0, T]$.

Remark 3.2. (i) $\mathcal{M}\left(C_{a, b}^{\prime}[0, T]\right)$ is a Banach algebra under the total variation norm where convolution is taken as the multiplication.

(ii) One can show that the correspondence $f \mapsto F$ is injective, carries convolution into pointwise multiplication and that $\mathcal{F}\left(C_{a, b}[0, T]\right)$ is a Banach algebra with norm

$$
\|F\|=\|f\|=\int_{C_{a, b}^{\prime}[0, T]}|d f(w)| .
$$

From now on, we will use the notation $(w, x)^{\sim}$ replaced by $\left\langle D_{t} w, x\right\rangle$. Then we have the following assertions.

(1) For each $w \in C_{a, b}^{\prime}[0, T]$, the random variable $x \mapsto(w, x)^{\sim}$ is Gaussian with mean $(w, a)_{C_{a, b}^{\prime}}$ and variance $\|w\|_{C_{a, b}^{\prime}}^{2}$.

(2) $(w, \alpha x)^{\sim}=\alpha(w, x)^{\sim}$ for any real number $\alpha, w \in C_{a, b}^{\prime}[0, T]$ and $x \in$ $C_{a, b}[0, T]$.

(3) If $\left\{w_{1}, w_{2}, \ldots, w_{n}\right\}$ is an orthonormal set in $C_{a, b}^{\prime}[0, T]$, then the random variables $\left(w_{j}, x\right)^{\sim}$ 's are independent.

We will explain the existence of generalized Feynman integrals of functionals in $\mathcal{F}\left(C_{a, b}[0, T]\right)$. Let $F$ be an element of $\mathcal{F}\left(C_{a, b}[0, T]\right)$ whose associated measure $f$ satisfies the condition

$$
\int_{C_{a, b}^{\prime}[0, T]} \exp \left\{\left|2 q_{0}\right|^{-\frac{1}{2}}\|w\|_{C_{a, b}^{\prime}}\|a\|_{C_{a, b}^{\prime}}\right\}|d f(w)|<+\infty
$$

for some $q_{0} \in \mathbb{R}-\{0\}$. Using the equation (3.9), Definition 2.2, the Fubini theorem and the equation (2.12), we see that for all real $q$ with $|q| \geq\left|q_{0}\right|$, the generalized analytic Feynman integral $E^{\operatorname{anf}_{q}}[F]$ of $F$ exists and is given by the formula

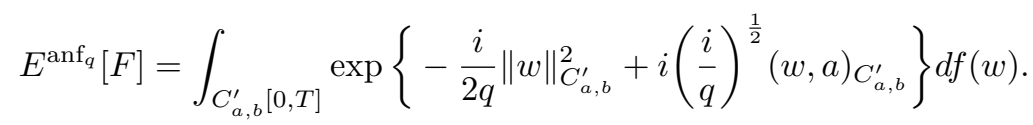

For more detail studies of existence of generalized Feynman integrals, see [7-11].

Throughout this section, for each $f \in \mathcal{M}\left(C_{a, b}^{\prime}[0, T]\right)$, we will use the notation

$$
d f_{\alpha q}^{\beta a}(w)=\exp \left\{i\left(\frac{i}{\alpha q}\right)^{\frac{1}{2}}(w, \beta a)_{C_{a, b}^{\prime}}\right\} d f(w) .
$$

The following theorems are due to Chang and Lee [10, 11]. 
Theorem 3.3. Let $q_{0}$ be a nonzero real number and let $F$ be an element of $\mathcal{F}\left(C_{a, b}[0, T]\right)$ whose associated measure $f$ satisfies the condition (3.10) above. Then for all $p \in[1,2]$ and real $q$ with $|q| \geq\left|q_{0}\right|$, the $L_{p}$ analytic $G F F T, T_{q}^{(p)}(F)$ of $F$, exists and is given by the formula

$$
T_{q}^{(p)}(F)(y)=\int_{C_{a, b}^{\prime}[0, T]} \exp \left\{i(w, y)^{\sim}-\frac{i}{2 q}\|w\|_{C_{a, b}^{\prime}}^{2}\right\} d f_{q}^{a}(w)
$$

for s-a.e. $y \in C_{a, b}[0, T]$. Furthermore, $T_{q}^{(p)}(F)$ is an element of $\mathcal{F}\left(C_{a, b}[0, T]\right)$ with associated measure $\phi$ defined by

$$
\phi(B)=\int_{B} \exp \left\{-\frac{i}{2 q}\|w\|_{C_{a, b}^{\prime}}^{2}\right\} d f_{q}^{a}(w)
$$

for $B \in \mathcal{B}\left(C_{a, b}^{\prime}[0, T]\right)$.

Remark 3.4. In Theorem 3.3 above, for all real $q$ with $|q| \geq\left|q_{0}\right|$ and $y \in$ $C_{a, b}[0, T]$,

$$
T_{q}^{(p)}(F)(y)=\int_{C_{a, b}[0, T]}^{\operatorname{anf}_{q}} F(y+x) d \mu(x), \quad 1 \leq p \leq 2 .
$$

In particular,

$$
T_{q}^{(p)}(F)(0)=\int_{C_{a, b}[0, T]}^{\operatorname{anf}_{q}} F(x) d \mu(x), \quad 1 \leq p \leq 2 .
$$

Theorem 3.5. Let $q_{0}$ and $F$ be as in Theorem 3.3. Then for all $p \in[1,2]$ and all real $q$ with $|q| \geq\left|q_{0}\right|$,

$$
\begin{aligned}
T_{-q}^{(p)}\left(T_{q}^{(p)}(F)\right)(y) & =\int_{C_{a, b}^{\prime}[0, T]} \exp \left\{i(w, y)^{\sim}+\frac{i}{\sqrt{|q / 2|}}(w, a)_{C_{a, b}^{\prime}}\right\} d f(w) \\
& =\int_{C_{a, b}^{\prime}[0, T]} \exp \left\{i(w, y)^{\sim}\right\} d f_{i|q / 2|}^{a}(w)
\end{aligned}
$$

for s-a.e. $y \in C_{a, b}[0, T]$. Furthermore, $T_{-q}^{(p)}\left(T_{q}^{(p)}(F)\right) \in \mathcal{F}\left(C_{a, b}[0, T]\right)$ and

$$
\left\|T_{-q}^{(p)}\left(T_{q}^{(p)}(F)\right)\right\|=\|F\| .
$$

In Theorem 3.5 above, let $a(t) \equiv 0$. Then $T_{-q}^{(p)}\left(T_{q}^{(p)}(F)\right)=F$ for s-a.e. $y \in C_{a, b}[0, T]$, that is, $T_{-q}^{(p)}$ is the inverse transform of $T_{q}^{(p)}$. For more details for the case $a(t) \equiv 0$, see $[3,13,14,16]$.

In our next theorem we obtain the $\mathrm{CP}$ of functionals in $\mathcal{F}\left(C_{a, b}[0, T]\right)$. The proof is given by a similar method of the proof of Theorem 3.2 in [4].

Theorem 3.6. Let $q_{0}$ be a nonzero real number and let $F$ and $G$ be elements of $\mathcal{F}\left(C_{a, b}[0, T]\right)$ whose associated measures $f$ and $g$ satisfy the condition

$$
\int_{C_{a, b}^{\prime}[0, T]} \exp \left\{\left|4 q_{0}\right|^{-\frac{1}{2}}\|w\|_{C_{a, b}^{\prime}}\|a\|_{C_{a, b}^{\prime}}\right\}[|d f(w)|+|d g(w)|]<+\infty .
$$


Then their $C P(F * G)_{q}$ exists for all real $q$ with $|q| \geq\left|q_{0}\right|$ and is given by the formula

$$
\begin{aligned}
(F * G)_{q}(y) & \int_{C_{a, b}^{\prime}[0, T]} \int_{C_{a, b}^{\prime}[0, T]} \exp \left\{\frac{i}{\sqrt{2}}\left(w_{1}+w_{2}, y\right)^{\sim}\right. \\
& \left.\quad-\frac{i}{4 q}\left\|w_{1}-w_{2}\right\|_{C_{a, b}^{\prime}}^{2}\right\} d f_{2 q}^{a}\left(w_{1}\right) d g_{2 q}^{-a}\left(w_{2}\right)
\end{aligned}
$$

for s-a.e. $y \in C_{a, b}[0, T]$. Furthermore, $(F * G)_{q}$ is an element of $\mathcal{F}\left(C_{a, b}[0, T]\right)$.

In Theorem 3.6 above, $(F * G)_{q}$ is expressible in the form

$$
(F * G)_{q}(y)=\int_{C_{a, b}^{\prime}[0, T]} \exp \left\{i(r, y)^{\sim}\right\} d\left(h \circ \psi^{-1}\right)(r)
$$

for s-a.e. $y \in C_{a, b}[0, T]$ where $\psi: C_{a, b}^{\prime}[0, T] \times C_{a, b}^{\prime}[0, T] \rightarrow C_{a, b}^{\prime}[0, T]$ is given by

$$
\psi\left(w_{1}+w_{2}\right)=\frac{1}{\sqrt{2}}\left(w_{1}+w_{2}\right)
$$

and $h$ is a complex Borel measure on $\mathcal{B}\left(C_{a, b}^{\prime}[0, T] \times C_{a, b}^{\prime}[0, T]\right)$ defined by

$$
h(B)=\int_{B} \exp \left\{-\frac{i}{4 q}\left\|w_{1}-w_{2}\right\|_{C_{a, b}^{\prime}}^{2}\right\} d f_{2 q}^{a}\left(w_{1}\right) d g_{2 q}^{-a}\left(w_{2}\right)
$$

for each $B \in \mathcal{B}\left(C_{a, b}^{\prime}[0, T] \times C_{a, b}^{\prime}[0, T]\right)$.

In our next theorem, we obtain the transform of the convolution product.

Theorem 3.7. Let $q_{0}$ be a nonzero real number and let $F$ and $G$ be elements of $\mathcal{F}\left(C_{a, b}[0, T]\right)$ whose associated measures $f$ and $g$ satisfy the condition

$$
\int_{C_{a, b}^{\prime}[0, T]} \exp \left\{2\left|4 q_{0}\right|^{-\frac{1}{2}}\|w\|_{C_{a, b}^{\prime}}\|a\|_{C_{a, b}^{\prime}}\right\}[|d f(w)|+|d g(w)|]<+\infty .
$$

Then for all $p \in[1,2]$ and all real $q$ with $|q| \geq\left|q_{0}\right|$,

$$
T_{q}^{(p)}\left((F * G)_{q}\right)(y)=T_{2 q}^{(p)}\left(T_{2 q}^{(p)}(F)\right)\left(\frac{y}{\sqrt{2}}\right) T_{2 q}^{(p)}\left(T_{2 q}^{(p)}(G(-\cdot))(-\cdot)\right)\left(\frac{y}{\sqrt{2}}\right)
$$

for s-a.e. $y \in C_{a, b}[0, T]$. Also, both of the expressions in (3.24) are given by the expression

$\int_{C_{a, b}^{\prime}[0, T]} \int_{C_{a, b}^{\prime}[0, T]} \exp \left\{\frac{i}{\sqrt{2}}\left(w_{1}+w_{2}, y\right)^{\sim}-\frac{i}{2 q}\left(\left\|w_{1}\right\|_{C_{a, b}^{\prime}}^{2}+\left\|w_{2}\right\|_{C_{a, b}^{\prime}}^{2}\right)\right\} d f_{2 q}^{2 a}\left(w_{1}\right) d g\left(w_{2}\right)$.

Proof. By using (2.8), (2.11), the Fubini theorem and (2.12), we have for all $\lambda>0$,

$$
T_{\lambda}\left((F * G)_{\lambda}\right)(y)=T_{2 \lambda}\left(T_{2 \lambda}(F)\right)\left(\frac{y}{\sqrt{2}}\right) T_{2 \lambda}\left(T_{2 \lambda}(G(-\cdot))(-\cdot)\right)\left(\frac{y}{\sqrt{2}}\right)
$$


for s-a.e. $y \in C_{a, b}[0, T]$. But both of the expressions on the right-hand side of equation (3.26) are analytic functions of $\lambda$ throughout $\mathbb{C}_{+}$, and are continuous functions of $\lambda$ on $\tilde{\mathbb{C}}_{+}$for all $y \in C_{a, b}[0, T]$. Furthermore, it is bounded on the region $\Gamma=\left\{\lambda \in \tilde{\mathbb{C}}_{+}:\left|\operatorname{Im}\left(\lambda^{-1 / 2}\right)\right| \leq 2\left|4 q_{0}\right|^{-1 / 2}\right\}$ under the condition (3.23). By using $(3.23), T_{q}^{(p)}\left((F * G)_{q}\right)$ exists for all real $q$ with $|q| \geq\left|q_{0}\right|$ and is given by (3.24) for all desired values of $p$ and $q$.

Theorem 3.8. Let $q_{0}$ be a nonzero real number and let $F$ and $G$ be elements of $\mathcal{F}\left(C_{a, b}[0, T]\right)$ whose associated measures $f$ and $g$ satisfy the condition

$$
\int_{C_{a, b}^{\prime}[0, T]} \exp \left\{3\left|4 q_{0}\right|^{-\frac{1}{2}}\|w\|_{C_{a, b}^{\prime}}\|a\|_{C_{a, b}^{\prime}}\right\}[|d f(w)|+|d g(w)|]<+\infty .
$$

Then for all $p \in[1,2]$ and all real $q$ with $|q| \geq\left|q_{0}\right|$,

$$
\begin{aligned}
(3.28) & \equiv \int_{C_{a, b}[0, T]}^{a n f_{-q}} T_{2 q}^{(p)}\left(T_{2 q}^{(p)}(F)\right)\left(\frac{y}{\sqrt{2}}\right) T_{2 q}^{(p)}\left(T_{2 q}^{(p)}(G(-\cdot))(-\cdot)\right)\left(\frac{y}{\sqrt{2}}\right) d \mu(y) \\
& =\int_{C_{a, b}[0, T]}^{a n f_{q}} T_{-2 q}^{(p)}\left(T_{2 q}^{(p)}(F)\right)\left(\frac{y}{\sqrt{2}}\right) T_{-2 q}^{(p)}\left(T_{2 q}^{(p)}(G)\right)\left(-\frac{y}{\sqrt{2}}\right) d \mu(y) .
\end{aligned}
$$

Also, both of the expressions in (3.28) are given by the expression

$$
\begin{aligned}
\int_{C_{a, b}^{\prime}[0, T]} \int_{C_{a, b}^{\prime}[0, T]} \exp \{- & \frac{i}{4 q}\left\|w_{1}-w_{2}\right\|_{C_{a, b}^{\prime}}^{2} \\
& \left.+i\left(\frac{-i}{2 q}\right)^{\frac{1}{2}}\left(w_{1}-w_{2}, a\right)_{C_{a, b}^{\prime}}\right\} d f_{2 q}^{2 a}\left(w_{1}\right) d g_{-2 q}^{2 a}\left(w_{2}\right) .
\end{aligned}
$$

Proof. Fix $p$ and $q$. Then for $\lambda>0$, using (3.24) and (3.12), we have

$$
\begin{aligned}
& \int_{C_{a, b}[0, T]} T_{q}^{(p)}\left((F * G)_{q}\right)(y / \sqrt{\lambda}) d \mu(y) \\
= & \int_{C_{a, b}^{\prime}[0, T]} \int_{C_{a, b}^{\prime}[0, T]} \exp \left\{-\frac{1}{4 \lambda}\left\|w_{1}+w_{2}\right\|_{C_{a, b}^{\prime}}^{2}+\frac{i}{\sqrt{2 \lambda}}\left(w_{1}+w_{2}, a\right)_{C_{a, b}^{\prime}}\right. \\
& \left.-\frac{i}{2 q}\left(\left\|w_{1}\right\|_{C_{a, b}^{\prime}}^{2}+\left\|w_{2}\right\|_{C_{a, b}^{\prime}}^{2}\right)+2 i\left(\frac{i}{2 q}\right)^{\frac{1}{2}}\left(w_{1}, a\right)_{C_{a, b}^{\prime}}\right\} d f\left(w_{1}\right) d g\left(w_{2}\right) .
\end{aligned}
$$

But the last expression of (3.30) is analytic through $\mathbb{C}_{+}$and is continuous on $\tilde{\mathbb{C}}_{+}$. Furthermore, it is bounded on the region $\Gamma=\left\{\lambda \in \tilde{\mathbb{C}}_{+}:\left|\operatorname{Im}\left(\lambda^{-1 / 2}\right)\right| \leq\right.$ 
$\left.3\left|4 q_{0}\right|^{-1 / 2}\right\}$ under condition (3.27). So letting $\lambda=-i(-q)=i q$, we have (3.31)

$$
\begin{aligned}
& \int_{C_{a, b}[0, T]}^{\operatorname{anf}_{-q}} T_{q}^{(p)}\left((F * G)_{q}\right)(y) d \mu(y) \\
= & \int_{C_{a, b}^{\prime}[0, T]} \int_{C_{a, b}^{\prime}[0, T]} \exp \left\{-\frac{i}{4 q}\left\|w_{1}-w_{2}\right\|_{C_{a, b}^{\prime}}^{2}+2 i\left(\frac{i}{2 q}\right)^{\frac{1}{2}}\left(w_{1}, a\right)_{C_{a, b}^{\prime}}\right. \\
& \left.+i\left(\frac{-i}{2 q}\right)^{\frac{1}{2}}\left(w_{1}+w_{2}, a\right)_{C_{a, b}^{\prime}}\right\} d f\left(w_{1}\right) d g\left(w_{2}\right) \\
= & \int_{C_{a, b}^{\prime}[0, T]} \int_{C_{a, b}^{\prime}[0, T]} \exp \left\{-\frac{i}{4 q}\left\|w_{1}-w_{2}\right\|_{C_{a, b}^{\prime}}^{2}\right. \\
& \left.+i\left(\frac{-i}{2 q}\right)^{\frac{1}{2}}\left(w_{1}-w_{2}, a\right)_{C_{a, b}^{\prime}}\right\} d f_{2 q}^{2 a}\left(w_{1}\right) d g_{-2 q}^{2 a}\left(w_{2}\right)
\end{aligned}
$$

for s-a.e. $y \in C_{a, b}[0, T]$.

On the other hand, using (3.12) and the Fubini theorem we have

$$
\begin{aligned}
& T_{-2 q}^{(p)}\left(T_{2 q}^{(p)}(F)\right)\left(\frac{y}{\sqrt{2}}\right) \\
= & \int_{C_{a, b}^{\prime}[0, T]} \exp \left\{\frac{i}{\sqrt{2}}\left(w_{1}, y\right)^{\sim}+i\left(\frac{-i}{2 q}\right)^{\frac{1}{2}}\left(w_{1}, a\right)_{C_{a, b}^{\prime}}\right\} d f_{2 q}^{a}\left(w_{1}\right)
\end{aligned}
$$

and

$$
\begin{aligned}
& T_{-2 q}^{(p)}\left(T_{2 q}^{(p)}(G)\right)\left(-\frac{y}{\sqrt{2}}\right) \\
= & \int_{C_{a, b}^{\prime}[0, T]} \exp \left\{-\frac{i}{\sqrt{2}}\left(w_{2}, y\right)^{\sim}+i\left(\frac{i}{2 q}\right)^{\frac{1}{2}}\left(w_{2}, a\right)_{C_{a, b}^{\prime}}\right\} d g_{-2 q}^{a}\left(w_{2}\right)
\end{aligned}
$$

for s-a.e. $y \in C_{a, b}[0, T]$. By using (3.32) and (3.33), we have for $\lambda>0$,

$$
\begin{aligned}
& \int_{C_{a, b}[0, T]} T_{-2 q}^{(p)}\left(T_{2 q}^{(p)}(F)\right)\left(\frac{y}{\sqrt{2 \lambda}}\right) T_{-2 q}^{(p)}\left(T_{2 q}^{(p)}(G)\right)\left(-\frac{y}{\sqrt{2 \lambda}}\right) d \mu(y) \\
= & \int_{C_{a, b}^{\prime}[0, T]} \int_{C_{a, b}^{\prime}[0, T]} \exp \left\{-\frac{1}{4 \lambda}\left\|w_{1}-w_{2}\right\|_{C_{a, b}^{\prime}}^{2}+\frac{i}{\sqrt{2 \lambda}}\left(w_{1}-w_{2}, a\right)_{C_{a, b}^{\prime}}\right. \\
+ & \left.i\left(\frac{-i}{2 q}\right)^{\frac{1}{2}}\left(w_{1}, a\right)_{C_{a, b}^{\prime}}+i\left(\frac{i}{2 q}\right)^{\frac{1}{2}}\left(w_{2}, a\right)_{C_{a, b}^{\prime}}\right\} d f_{2 q}^{a}\left(w_{1}\right) d g_{-2 q}^{a}\left(w_{2}\right) .
\end{aligned}
$$

But the last expression above is an analytic function of $\lambda$ throughout $\mathbb{C}_{+}$and is continuous throughout on $\tilde{\mathbb{C}}_{+}$. Also, it is bounded on the region $\Gamma=\{\lambda \in$ 
$\left.\tilde{\mathbb{C}}_{+}:\left|\operatorname{Im}\left(\lambda^{-1 / 2}\right)\right| \leq 3\left|4 q_{0}\right|^{-1 / 2}\right\}$. Letting $\lambda=-i q$ we have

$$
\begin{aligned}
& \int_{C_{a, b}[0, T]}^{\operatorname{anf}_{q}} T_{-2 q}^{(p)}\left(T_{2 q}^{(p)}(F)\right)\left(\frac{y}{\sqrt{2}}\right) T_{-2 q}^{(p)}\left(T_{2 q}^{(p)}(G)\right)\left(-\frac{y}{\sqrt{2}}\right) d \mu(y) \\
= & \int_{C_{a, b}^{\prime}[0, T]} \int_{C_{a, b}^{\prime}[0, T]} \exp \left\{-\frac{i}{4 q}\left\|w_{1}-w_{2}\right\|_{C_{a, b}^{\prime}}^{2}\right. \\
& \left.+i\left(\frac{-i}{2 q}\right)^{\frac{1}{2}}\left(w_{1}-w_{2}, a\right)_{C_{a, b}^{\prime}}\right\} d f_{2 q}^{2 a}\left(w_{1}\right) d g_{-2 q}^{2 a}\left(w_{2}\right) .
\end{aligned}
$$

Now (3.31) and (3.35) together yield (3.28) .

\section{Transforms and convolutions of functionals in $\mathcal{F}_{A_{1}, A_{2}}$}

Let $A$ be a nonnegative self-adjoint operator on $C_{a, b}^{\prime}[0, T]$ and $f$ any finite complex measure. Then the functional

$$
F(x)=\int_{C_{a, b}^{\prime}[0, T]} \exp \left\{i\left(A^{\frac{1}{2}} w, x\right)^{\sim}\right\} d f(w)
$$

belongs to $\mathcal{F}\left(C_{a, b}[0, T]\right)$ because it can be rewritten as

$$
\int_{C_{a, b}^{\prime}[0, T]} \exp \left\{i(w, x)^{\sim}\right\} d \nu(w)
$$

for $\nu=f \circ\left(A^{1 / 2}\right)^{-1}$. Let $A$ be self-adjoint but not nonnegative. Then $A$ has the form

$$
A=A^{+}-A^{-}
$$

and both $A^{+}$and $A^{-}$are bounded nonnegative self-adjoint operators.

In this section we will get expressions of the generalized Feynman integral, the GFFT and the CP when $A$ is no longer required to be nonnegative or even self-adjoint. In order to widen the scope of the analytic continuation technique to treat such cases, we will present definitions here in a slightly modified form.

Given two $\mathbb{C}$-valued measurable functions $F$ and $G$ on $C_{a, b}^{2}[0, T], F$ is said to be equal to $G$ scale almost everywhere(s-a.e.) if for each $\rho_{1}, \rho_{2}>0, \mu\left(\left\{\left(x_{1}, x_{2}\right)\right.\right.$ $\left.\left.\in C_{a, b}^{2}[0, T]: F\left(\rho_{1} x_{1}, \rho_{2} x_{2}\right) \neq G\left(\rho_{1} x_{1}, \rho_{2}, x_{2}\right)\right\}\right)=0$. We write that $F \approx G$ if $F=G$ s-a.e..

Definition 4.1. Let $\mathbb{C}_{+}^{2}=\left\{\vec{\lambda}=\left(\lambda_{1}, \lambda_{2}\right) \in \mathbb{C}^{2}: \operatorname{Re}\left(\lambda_{j}\right)>0\right.$ for $\left.j=1,2\right\}$ and let $\tilde{\mathbb{C}}_{+}^{2}=\left\{\vec{\lambda}=\left(\lambda_{1}, \lambda_{2}\right) \in \mathbb{C}^{2}: \lambda_{j} \neq 0\right.$ and $\operatorname{Re}\left(\lambda_{j}\right) \geq 0$ for $\left.j=1,2\right\}$. Let $F: C_{a, b}^{2}[0, T] \rightarrow \mathbb{C}$ be a measurable functional such that for each $\lambda_{1}, \lambda_{2}>0$, the function space integral

$$
J\left(\lambda_{1}, \lambda_{2}\right)=\int_{C_{a, b}^{2}[0, T]} F\left(\lambda_{1}^{-1 / 2} x_{1}, \lambda_{2}^{-1 / 2} x_{2}\right) d \mu\left(x_{1}, x_{2}\right)
$$


exists. If there exists a function $J^{*}\left(\lambda_{1}, \lambda_{2}\right)$ analytic in $\mathbb{C}_{+}^{2}$ such that $J^{*}\left(\lambda_{1}, \lambda_{2}\right)$ $=J\left(\lambda_{1}, \lambda_{2}\right)$ for all $\lambda_{1}, \lambda_{2}>0$, then $J^{*}\left(\lambda_{1}, \lambda_{2}\right)$ is defined to be the analytic function space integral of $F$ over $C_{a, b}^{2}[0, T]$ with parameter $\vec{\lambda}=\left(\lambda_{1}, \lambda_{2}\right)$, and for $\vec{\lambda} \in \mathbb{C}_{+}^{2}$ we write

$$
E^{\mathrm{an}_{\vec{\lambda}}}[F] \equiv E_{\vec{x}}^{\operatorname{an}_{\vec{\lambda}}}\left[F\left(x_{1}, x_{2}\right)\right] \equiv \int_{C_{a, b}^{2}[0, T]}^{\mathrm{an}_{\vec{\lambda}}} F\left(x_{1}, x_{2}\right) d(\mu \times \mu)\left(x_{1}, x_{2}\right)=J^{*}(\vec{\lambda}) .
$$

Let $q_{1}$ and $q_{2}$ be nonzero real numbers. Let $F$ be a functional such that $E^{\operatorname{an}_{\bar{\lambda}}}[F]$ exists for all $\vec{\lambda} \in \mathbb{C}_{+}^{2}$. If the following limit exists, we call it the generalized analytic Feynman integral of $F$ with parameter $\vec{q}=\left(q_{1}, q_{2}\right)$ and we write

$$
\begin{aligned}
E^{\operatorname{anf}_{\vec{q}}}[F] & \equiv E_{\vec{x}}^{\operatorname{anf}_{\vec{q}}}\left[F\left(x_{1}, x_{2}\right)\right] \\
& \equiv \int_{C_{a, b}^{2}[0, T]}^{\operatorname{anf}_{\vec{q}}} F\left(x_{1}, x_{2}\right) d(\mu \times \mu)\left(x_{1}, x_{2}\right)=\lim _{\vec{\lambda} \rightarrow-i \vec{q}} E^{\operatorname{an}_{\vec{\lambda}}[F],}
\end{aligned}
$$

where $\vec{\lambda} \rightarrow-i \vec{q}=\left(-i q_{1},-i q_{2}\right)$ through values in $\mathbb{C}_{+}^{2}$.

Definition 4.2. Let $q_{1}$ and $q_{2}$ be nonzero real numbers. For $\vec{\lambda}=\left(\lambda_{1}, \lambda_{2}\right) \in \mathbb{C}_{+}^{2}$ and $\left(y_{1}, y_{2}\right) \in C_{a, b}^{2}[0, T]$, let

$$
T_{\vec{\lambda}}(F)\left(y_{1}, y_{2}\right)=E_{\vec{x}}^{\mathrm{an}_{\vec{\lambda}}}\left[F\left(y_{1}+x_{1}, y_{2}+x_{2}\right)\right] .
$$

For $p \in(1,2]$, we define the $L_{p}$ analytic $\operatorname{GFFT}, T_{\vec{q}}^{(p)}(F)$ of $F$, by the formula $\left(\vec{\lambda} \in \mathbb{C}_{+}^{2}\right)$

$$
T_{\vec{q}}^{(p)}(F)\left(y_{1}, y_{2}\right)=\text { l.i.m. } \cdot \vec{\lambda} \rightarrow-i \vec{q} T_{\vec{\lambda}}(F)\left(y_{1}, y_{2}\right)
$$

if it exists; i.e., for each $\rho_{1}, \rho_{2}>0$,

$\lim _{\vec{\lambda} \rightarrow-i \vec{q}} \int_{C_{a, b}^{2}[0, T]}\left|T_{\vec{\lambda}}(F)\left(\rho_{1} y_{1}, \rho_{2} y_{2}\right)-T_{\vec{q}}^{(p)}(F)\left(\rho_{1} y_{1}, \rho_{2} y_{2}\right)\right|^{p^{\prime}} d(\mu \times \mu)\left(y_{1}, y_{2}\right)=0$,

where $1 / p+1 / p^{\prime}=1$. We define the $L_{1}$ analytic GFFT, $T_{\vec{q}}^{(1)}(F)$ of $F$, by the formula $\left(\vec{\lambda} \in \mathbb{C}_{+}^{2}\right)$

$$
T_{\vec{q}}^{(1)}(F)\left(y_{1}, y_{2}\right)=\lim _{\vec{\lambda} \rightarrow-i \vec{q}} T_{\vec{\lambda}}(F)\left(y_{1}, y_{2}\right)
$$

if it exists.

We note that for $1 \leq p \leq 2, T_{\vec{q}}^{(p)}(F)$ is defined only s-a.e.. We also note that if $T_{\vec{q}}^{(p)}(F)$ exists and if $F \approx G$, then $T_{\vec{q}}^{(p)}(G)$ exists and $T_{\vec{q}}^{(p)}(G) \approx T_{\vec{q}}^{(p)}(F)$.

Next we give the definition of the $\mathrm{CP}$ on $C_{a, b}^{2}[0, T]$. 
Definition 4.3. Let $F$ and $G$ be measurable functionals on $C_{a, b}^{2}[0, T]$. For $\vec{\lambda} \in \tilde{\mathbb{C}}_{+}^{2}$, we define their $\mathrm{CP}(F * G)_{\vec{\lambda}}$ (if it exists) by

$$
\begin{aligned}
& (F * G)_{\vec{\lambda}}\left(y_{1}, y_{2}\right) \\
= & \left\{\begin{aligned}
E_{\vec{x}}^{\operatorname{an}_{\vec{\lambda}}}\left[F\left(\frac{y_{1}+x_{1}}{\sqrt{2}}, \frac{y_{2}+x_{2}}{\sqrt{2}}\right) G\left(\frac{y_{1}-x_{1}}{\sqrt{2}}, \frac{y_{2}-x_{2}}{\sqrt{2}}\right)\right], \quad \vec{\lambda} \in \mathbb{C}_{+} \\
E_{\vec{x}}^{\operatorname{anf}_{\vec{q}}}\left[F\left(\frac{y_{1}+x_{1}}{\sqrt{2}}, \frac{y_{2}+x_{2}}{\sqrt{2}}\right) G\left(\frac{y_{1}-x_{1}}{\sqrt{2}}, \frac{y_{2}-x_{2}}{\sqrt{2}}\right)\right], \\
\vec{\lambda}=-i \vec{q}=\left(-i q_{1},-i q_{2}\right), q_{1}, q_{2} \in \mathbb{R}-\{0\} .
\end{aligned}\right.
\end{aligned}
$$

Definition 4.4. Let $A_{1}$ and $A_{2}$ be bounded, nonnegative self-adjoint operators on $C_{a, b}^{\prime}[0, T]$. The Banach algebra $\mathcal{F}_{A_{1}, A_{2}}$ consists of those functionals $F$ on $C_{a, b}^{2}[0, T]$ expressible in the form

$$
F\left(x_{1}, x_{2}\right)=\int_{C_{a, b}^{\prime}[0, T]} \exp \left\{i\left(A_{1}^{\frac{1}{2}} w, x_{1}\right)^{\sim}+i\left(A_{2}^{\frac{1}{2}} w, x_{2}\right)^{\sim}\right\} d f(w)
$$

for s-a.e. $\left(x_{1}, x_{2}\right) \in C_{a, b}^{2}[0, T]$, where the associated measure $f$ is an element $\mathcal{M}\left(C_{a, b}^{\prime}[0, T]\right)$.

Remark 4.5. In Definition 4.4 above, let $A_{1}$ be the identity operator on $C_{a, b}^{\prime}[0, T]$ and $A_{2} \equiv 0$. Then $\mathcal{F}_{A_{1}, A_{2}}$ is essentially the Fresnel type class $\mathcal{F}\left(C_{a, b}[0, T]\right)$ which was defined in Section 3 , and for real $q_{j}, j=1,2$,

$$
E_{\vec{x}}^{\operatorname{anf}_{\vec{q}}}\left[F\left(x_{1}, x_{2}\right)\right]=\int_{C_{a, b}[0, T]}^{\operatorname{anf}_{q_{1}}} F_{0}\left(x_{1}\right) d \mu\left(x_{1}\right)
$$

if it exists, where $F_{0}\left(x_{1}\right)=F\left(x_{1}, x_{2}\right)$ for all $\left(x_{1}, x_{2}\right) \in C_{a, b}^{2}[0, T]$ and

$$
\int_{C_{a, b}[0, T]}^{\operatorname{anf}_{q_{1}}} F_{0}\left(x_{1}\right) d \mu\left(x_{1}\right)
$$

means the generalized analytic Feynman integral on $C_{a, b}[0, T]$ which was defined in Section 2 above.

Let $A_{j}: C_{a, b}^{\prime}[0, T] \rightarrow C_{a, b}^{\prime}[0, T], j=1,2$ be nonnegative self-adjoint operators. Throughout this section, for each $f \in \mathcal{M}\left(C_{a, b}^{\prime}[0, T]\right)$, we will use the notation

$$
d f_{\alpha \vec{q}}^{\vec{A}, \beta a}(w)=\exp \left\{i\left(\frac{i}{\alpha q_{1}}\right)^{\frac{1}{2}}\left(A_{1}^{\frac{1}{2}} w, \beta a\right)_{C_{a, b}^{\prime}}+i\left(\frac{i}{\alpha q_{2}}\right)^{\frac{1}{2}}\left(A_{2}^{\frac{1}{2}} w, \beta a\right)_{C_{a, b}^{\prime}}\right\} d f(w) .
$$

In our next theorem, we obtain the $L_{p}$ analytic $\operatorname{GFFT} T_{\vec{q}}^{(p)}(F)$ of a functional $F$ in $\mathcal{F}_{A_{1}, A_{2}}$.

Theorem 4.6. Let $q_{0}$ be a nonzero real number and let $F$ be an element of $\mathcal{F}_{A_{1}, A_{2}}$ whose associated measure $f$ satisfies the condition

$$
\int_{C_{a, b}^{\prime}[0, T]} \exp \left\{\left|2 q_{0}\right|^{-\frac{1}{2}}\left(\left\|A_{1}^{\frac{1}{2}} w\right\|_{C_{a, b}^{\prime}}+\left\|A_{2}^{\frac{1}{2}} w\right\|_{C_{a, b}^{\prime}}\right)\|a\|_{C_{a, b}^{\prime}}\right\} d f(w)<+\infty .
$$


Then for all $p \in[1,2]$ and all real $q_{j}$ with $\left|q_{j}\right| \geq\left|q_{0}\right|, j=1,2$, the $L_{p}$ analytic GFFT, $T_{\vec{q}}^{(p)}(F)$ of $F$ exists and is given by the formula

$$
\begin{aligned}
T_{\vec{q}}^{(p)}(F)\left(y_{1}, y_{2}\right)= & \int_{C_{a, b}^{\prime}[0, T]} \exp \left\{i\left(A_{1}^{\frac{1}{2}} w, y_{1}\right)^{\sim}+i\left(A_{2}^{\frac{1}{2}} w, y_{2}\right)^{\sim}\right. \\
& \left.-\frac{i}{2 q_{1}}\left\|A_{1}^{\frac{1}{2}} w\right\|_{C_{a, b}^{\prime}}^{2}-\frac{i}{2 q_{2}}\left\|A_{2}^{\frac{1}{2}} w\right\|_{C_{a, b}^{\prime}}^{2}\right\} d f_{\vec{q}}^{\vec{A}, a}(w)
\end{aligned}
$$

for s-a.e. $\left(y_{1}, y_{2}\right) \in C_{a, b}^{2}[0, T]$. Furthermore, $T_{\vec{q}}^{(p)}(F)$ is an element of $\mathcal{F}_{A_{1}, A_{2}}$ with associated measure $\phi$ defined by

$$
\phi(B)=\int_{B} \exp \left\{-\frac{i}{2 q_{1}}\left\|A_{1}^{\frac{1}{2}} w\right\|_{C_{a, b}^{\prime}}^{2}-\frac{i}{2 q_{2}}\left\|A_{2}^{\frac{1}{2}} w\right\|_{C_{a, b}^{\prime}}^{2}\right\} d f_{\vec{q}}^{\vec{A}, a}(w)
$$

for $B \in \mathcal{B}\left(C_{a, b}^{\prime}[0, T]\right)$.

Proof. For $\lambda_{j}>0, j=1,2$ and s-a.e. $\left(y_{1}, y_{2}\right) \in C_{a, b}^{2}[0, T]$, using the equation (4.4), the Fubini theorem and the equation (2.12), we have

$$
\begin{aligned}
& T_{\vec{\lambda}}(F)\left(y_{1}, y_{2}\right) \\
&= E_{\vec{x}}\left[F\left(y_{1}+\lambda_{1}^{-\frac{1}{2}} x_{1}, y_{2}+\lambda_{2}^{-\frac{1}{2}} x_{2}\right)\right] \\
&= \int_{C_{a, b}^{\prime}[0, T]} E_{\vec{x}}\left[\operatorname { e x p } \left\{i\left(A_{1}^{\frac{1}{2}} w, y_{1}\right)^{\sim}+i \lambda_{1}^{-\frac{1}{2}}\left(A_{1}^{\frac{1}{2}} w, x_{1}\right)^{\sim}\right.\right. \\
&\left.\left.\left.+i\left(A_{2}^{\frac{1}{2}} w, y_{2}\right)^{\sim}+i \lambda_{2}^{-\frac{1}{2}}\left(A_{2}^{\frac{1}{2}} w, x_{2}\right)^{\sim}\right)\right\}\right] d f(w) \\
&= \int_{C_{a, b}^{\prime}[0, T]} \exp \left\{i\left(A_{1}^{\frac{1}{2}} w, y_{1}\right)^{\sim}+i\left(A_{2}^{\frac{1}{2}} w, y_{2}\right)^{\sim}\right. \\
& \quad-\frac{1}{2 \lambda_{1}}\left\|A_{1}^{\frac{1}{2}} w\right\|_{C_{a, b}^{\prime}}^{2}-\frac{1}{2 \lambda_{2}}\left\|A_{2}^{\frac{1}{2}} w\right\|_{C_{a, b}^{\prime}}^{2} \\
&\left.\quad+\frac{i}{\sqrt{\lambda_{1}}}\left(A_{1}^{\frac{1}{2}} w, a\right)_{C_{a, b}^{\prime}}+\frac{i}{\sqrt{\lambda_{2}}}\left(A_{2}^{\frac{1}{2}} w, a\right)_{C_{a, b}^{\prime}}\right\} d f(w) .
\end{aligned}
$$

But the last expression above is analytic through $\mathbb{C}_{+}^{2}$ and is continuous on $\tilde{\mathbb{C}}_{+}^{2}$. Also, it is bounded on the region $\Gamma=\left\{\vec{\lambda}=\left(\lambda_{1}, \lambda_{2}\right) \in \tilde{\mathbb{C}}_{+}^{2}:\left|\operatorname{Im}\left(\lambda_{j}^{-1 / 2}\right)\right| \leq\right.$ $\left.\left|2 q_{0}\right|^{-1 / 2}, j=1,2\right\}$. Thus the equation (4.10) is established.

Let $\phi$ be a set function on $\mathcal{B}\left(C_{a, b}^{\prime}[0, T]\right)$ defined by the equation (4.11). By using the condition (4.9) we see that

$$
\begin{aligned}
\|\phi\|= & \int_{C_{a, b}^{\prime}[0, T]}\left|d f_{\vec{q}}^{\vec{A}, a}(w)\right| \\
\leq & \int_{C_{a, b}^{\prime}[0, T]} \exp \left\{\frac{1}{\sqrt{\left|2 q_{0}\right|}}\left\|A_{1}^{\frac{1}{2}} w\right\|_{C_{a, b}^{\prime}}\|a\|_{C_{a, b}^{\prime}}\right. \\
& \left.\quad+\frac{1}{\sqrt{\left|2 q_{0}\right|}}\left\|A_{2}^{\frac{1}{2}} w\right\|_{C_{a, b}^{\prime}}\|a\|_{C_{a, b}^{\prime}}\right\}|d f(w)|<+\infty .
\end{aligned}
$$


Hence we have the desired result.

Let $A$ be self-adjoint but not nonnegative. Then $A$ has the form (4.1). Let $F \in \mathcal{F}_{A_{+}, A_{-}}$. Suppose that the associated measure $f$ of $F$ satisfies condition (4.9) with $A_{1}$ and $A_{2}$ replaced with $A_{+}$and $A_{-}$, respectively. Then for $\vec{q}=$ $(q,-q)$ with $q \in \mathbb{R}-\{0\}$ and $|q| \geq\left|q_{0}\right|$,

$$
\begin{aligned}
& T_{\vec{q}}^{(p)}(F)\left(y_{1}, y_{2}\right) \\
= & \int_{C_{a, b}^{\prime}[0, T]} \exp \left\{i\left(A_{+}^{\frac{1}{2}} w, y_{1}\right)^{\sim}+i\left(A_{-}^{\frac{1}{2}} w, y_{2}\right)^{\sim}-\frac{i}{2 q}\left\|A^{\frac{1}{2}} w\right\|_{C_{a, b}^{\prime}}^{2}\right\} d f_{\vec{q}}^{\left(A_{+}, A_{-}\right), a}(w)
\end{aligned}
$$

and

$$
E^{\operatorname{anf}_{\vec{q}}[F]}=T_{\vec{q}}^{(p)}(F)(0,0)=\int_{C_{a, b}^{\prime}[0, T]} \exp \left\{-\frac{i}{2 q}\left\|A^{\frac{1}{2}} w\right\|_{C_{a, b}^{\prime}}^{2}\right\} d f_{\vec{q}}^{\left(A_{+}, A_{-}\right), a}(w) .
$$

Moreover, if $a(t) \equiv 0$, then

$$
\begin{aligned}
& T_{\vec{q}}^{(p)}(F)\left(y_{1}, y_{2}\right) \\
= & \int_{C_{a, b}^{\prime}[0, T]} \exp \left\{i\left(A_{+}^{\frac{1}{2}} w, y_{1}\right)^{\sim}+i\left(A_{-}^{\frac{1}{2}} w, y_{2}\right)^{\sim}-\frac{i}{2 q}\left\|A^{\frac{1}{2}} w\right\|_{C_{a, b}^{\prime}}^{2}\right\} d f(w)
\end{aligned}
$$

and

$$
E^{\operatorname{anf}_{\vec{q}}[F]}=T_{\vec{q}}^{(p)}(F)(0,0)=\int_{C_{a, b}^{\prime}[0, T]} \exp \left\{-\frac{i}{2 q}\left\|A^{\frac{1}{2}} w\right\|_{C_{a, b}^{\prime}}^{2}\right\} d f(w) .
$$

In our next theorem, we obtain the $\mathrm{CP}$ of functionals in $\mathcal{F}_{A_{1}, A_{2}}$.

Theorem 4.7. Let $q_{0}$ be a nonzero real number and let $F$ and $G$ be elements of $\mathcal{F}_{A_{1}, A_{2}}$ whose associated measures $f$ and $g$ satisfy the condition

$$
\begin{aligned}
\int_{C_{a, b}^{\prime}[0, T]} \exp \left\{| 4 q _ { 0 } | ^ { - \frac { 1 } { 2 } } \left(\left\|A_{1}^{\frac{1}{2}} w\right\|_{C_{a, b}^{\prime}}\right.\right. \\
\left.\left.+\left\|A_{2}^{\frac{1}{2}} w\right\|_{C_{a, b}^{\prime}}\right)\|a\|_{C_{a, b}^{\prime}}\right\}[|d f(w)|+|d g(w)|]<+\infty .
\end{aligned}
$$

Then their $C P(F * G)_{\vec{q}}$ exists for all real $q_{j}$ with $\left|q_{j}\right| \geq\left|q_{0}\right|, j=1,2$ and is given by the formula

$$
\begin{aligned}
& (F * G)_{\vec{q}}\left(y_{1}, y_{2}\right) \\
& =\int_{C_{a, b}^{\prime}[0, T]} \int_{C_{a, b}^{\prime}[0, T]} \exp \left\{\frac{i}{\sqrt{2}}\left(A_{1}^{\frac{1}{2}}\left(w_{1}+w_{2}\right), y_{1}\right)^{\sim}\right. \\
& \quad+\frac{i}{\sqrt{2}}\left(A_{2}^{\frac{1}{2}}\left(w_{1}+w_{2}\right), y_{2}\right)^{\sim}-\frac{i}{4 q_{1}}\left\|A_{1}^{\frac{1}{2}}\left(w_{1}-w_{2}\right)\right\|_{C_{a, b}^{\prime}}^{2}
\end{aligned}
$$




$$
\left.-\frac{i}{4 q_{2}}\left\|A_{2}^{\frac{1}{2}}\left(w_{1}-w_{2}\right)\right\|_{C_{a, b}^{\prime}}^{2}\right\} d f_{2 \vec{q}}^{\vec{A}, a}\left(w_{1}\right) d g_{2 \vec{q}}^{\vec{A},-a}\left(w_{2}\right)
$$

for s-a.e. $\left(y_{1}, y_{2}\right) \in C_{a, b}^{2}[0, T]$. Furthermore, $(F * G)_{\vec{q}}$ is an element of $\mathcal{F}_{A_{1}, A_{2}}$.

Proof. By using (4.7), the Fubini theorem and (2.12), we have for $\lambda_{j}>0$, $j=1,2$

$$
\begin{gathered}
(F * G)_{\vec{\lambda}}\left(y_{1}, y_{2}\right) \\
=\int_{C_{a, b}^{\prime}[0, T]} \int_{C_{a, b}^{\prime}[0, T]} \exp \left\{\frac{i}{\sqrt{2}}\left(A_{1}^{\frac{1}{2}}\left(w_{1}+w_{2}\right), y_{1}\right)^{\sim}\right. \\
+\frac{i}{\sqrt{2}}\left(A_{2}^{\frac{1}{2}}\left(w_{1}+w_{2}\right), y_{2}\right)^{\sim}-\frac{1}{4 \lambda_{1}}\left\|A_{1}^{\frac{1}{2}}\left(w_{1}-w_{2}\right)\right\|_{C_{a, b}^{\prime}}^{2} \\
-\frac{1}{4 \lambda_{2}}\left\|A_{2}^{\frac{1}{2}}\left(w_{1}-w_{2}\right)\right\|_{C_{a, b}^{\prime}}^{2}+i\left(\frac{1}{2 \lambda_{1}}\right)^{\frac{1}{2}}\left(A_{1}^{\frac{1}{2}}\left(w_{1}-w_{2}\right), a\right)_{C_{a, b}^{\prime}} \\
\left.+i\left(\frac{1}{2 \lambda_{2}}\right)^{\frac{1}{2}}\left(A_{2}^{\frac{1}{2}}\left(w_{1}-w_{2}\right), a\right)_{C_{a, b}^{\prime}}\right\} d f\left(w_{1}\right) d g\left(w_{2}\right)
\end{gathered}
$$

for s-a.e. $\left(y_{1}, y_{2}\right) \in C_{a, b}^{2}[0, T]$. But the last expression above is analytic throughout $\mathbb{C}_{+}$, is continuous on $\tilde{\mathbb{C}}_{+}$, and is bounded on the region $\Gamma=\left\{\vec{\lambda}=\left(\lambda_{1}, \lambda_{2}\right) \in\right.$ $\left.\tilde{\mathbb{C}}_{+}^{2}:\left|\operatorname{Im}\left(\lambda_{j}^{-1 / 2}\right)\right| \leq\left|4 q_{0}\right|^{-1 / 2}, j=1,2\right\}$. Thus letting $\vec{\lambda}=-i \vec{q}$ and using a simple calculation, we have the equation (4.19) above.

Let a set function $h: \mathcal{B}\left(C_{a, b}^{\prime}[0, T] \times C_{a, b}^{\prime}[0, T]\right) \rightarrow \mathbb{C}$ be defined by

$$
\begin{aligned}
h(B)=\int_{B} \exp & \left\{-\frac{i}{4 q_{1}}\left\|A_{1}^{\frac{1}{2}}\left(w_{1}-w_{2}\right)\right\|_{C_{a, b}^{\prime}}^{2}\right. \\
& \left.-\frac{i}{4 q_{2}}\left\|A_{2}^{\frac{1}{2}}\left(w_{1}-w_{2}\right)\right\|_{C_{a, b}^{\prime}}^{2}\right\} d f_{2 \vec{q}}^{\vec{A}, a}\left(w_{1}\right) d g_{2 \vec{q}}^{\vec{A},-a}\left(w_{2}\right)
\end{aligned}
$$

for each $B \in \mathcal{B}\left(C_{a, b}^{\prime}[0, T] \times C_{a, b}^{\prime}[0, T]\right)$. Then $h$ is a complex Borel measure on $\mathcal{B}\left(C_{a, b}^{\prime}[0, T] \times C_{a, b}^{\prime}[0, T]\right)$. Now we define a function $\psi: C_{a, b}^{\prime}[0, T] \times C_{a, b}^{\prime}[0, T] \rightarrow$ $C_{a, b}^{\prime}[0, T]$ by $\psi\left(w_{1}, w_{2}\right)=\left(w_{1}+w_{2}\right) / \sqrt{2}$. Then $\psi$ is continuous and so it is Borel measurable. Let $\tilde{h}=h \circ \psi^{-1}$. By the condition (4.18) above, we see that for real $q_{j}$ with $\left|q_{j}\right| \geq\left|q_{0}\right|, j=1,2$,

$$
\begin{aligned}
\|\tilde{h}\|= & \int_{C_{a, b}^{\prime}[0, T]} \int_{C_{a, b}^{\prime}[0, T]}\left|\operatorname{dh}\left(w_{1}, w_{2}\right)\right| \\
\leq & \int_{C_{a, b}^{\prime}[0, T]} \int_{C_{a, b}^{\prime}[0, T]} \mid \exp \left\{-\frac{i}{4 q_{1}}\left\|A_{1}^{\frac{1}{2}}\left(w_{1}-w_{2}\right)\right\|_{C_{a, b}^{\prime}}^{2}\right. \\
& \quad-\frac{i}{4 q_{2}}\left\|A_{2}^{\frac{1}{2}}\left(w_{1}-w_{2}\right)\right\|_{C_{a, b}^{\prime}}^{2}+i\left(\frac{i}{2 q_{1}}\right)^{\frac{1}{2}}\left(A_{1}^{\frac{1}{2}}\left(w_{1}-w_{2}\right), a\right)_{C_{a, b}^{\prime}}
\end{aligned}
$$




$$
\begin{aligned}
& \left.+i\left(\frac{i}{2 q_{2}}\right)^{\frac{1}{2}}\left(A_{2}^{\frac{1}{2}}\left(w_{1}-w_{2}\right), a\right)_{C_{a, b}^{\prime}}\right\}|| d f\left(w_{1}\right)|| d g\left(w_{2}\right) \mid \\
\leq & \int_{C_{a, b}^{\prime}[0, T]} \exp \left\{\frac{1}{\sqrt{\left|4 q_{0}\right|}}\left(\left\|A_{1}^{\frac{1}{2}} w_{1}\right\|_{C_{a, b}^{\prime}}+\left\|A_{2}^{\frac{1}{2}} w_{1}\right\|_{C_{a, b}^{\prime}}\right)\|a\|_{C_{a, b}^{\prime}}\right\}\left|d f\left(w_{1}\right)\right| \\
& \cdot \int_{C_{a, b}^{\prime}[0, T]} \exp \left\{\frac{1}{\sqrt{\left|4 q_{0}\right|}}\left(\left\|A_{1}^{\frac{1}{2}} w_{2}\right\|_{C_{a, b}^{\prime}}+\left\|A_{2}^{\frac{1}{2}} w_{2}\right\|_{C_{a, b}^{\prime}}\right)\|a\|_{C_{a, b}^{\prime}}\right\}\left|d g\left(w_{2}\right)\right| \\
<\infty . &
\end{aligned}
$$

Hence $\tilde{h}=h \circ \psi^{-1}$ belongs to $\mathcal{M}\left(C_{a, b}^{\prime}[0, T]\right)$ and

$$
(F * G)_{\vec{q}}\left(y_{1}, y_{2}\right)=\int_{C_{a, b}^{\prime}[0, T]} \exp \left\{i\left(A_{1}^{\frac{1}{2}} r, y_{1}\right)^{\sim}+i\left(A_{2}^{\frac{1}{2}} r, y_{2}\right)^{\sim}\right\} d \tilde{h}(r)
$$

for s-a.e. $\left(y_{1}, y_{2}\right) \in C_{a, b}^{2}[0, T]$. Hence $(F * G)_{\vec{q}}$ exists and is given by (4.19) for all real $q_{j}$ with $\left|q_{j}\right| \geq\left|q_{0}\right|$ and it belongs to $\mathcal{F}_{A_{1}, A_{2}}$.

In next two theorems, we also give some relationships of the GFFT and the $\mathrm{CP}$ of functionals in $\mathcal{F}_{A_{1}, A_{2}}$ without proofs.

Theorem 4.8. Let $q_{0}$ be a nonzero real number and let $F$ and $G$ be elements of $\mathcal{F}_{A_{1}, A_{2}}$ whose associated measures $f$ and $g$ satisfy the condition (4.24)

$$
\begin{aligned}
\int_{C_{a, b}^{\prime}[0, T]} \exp \left\{2 | 4 q _ { 0 } | ^ { - \frac { 1 } { 2 } } \left(\left\|A_{1}^{\frac{1}{2}} w\right\|_{C_{a, b}^{\prime}}\right.\right. \\
\left.\left.+\left\|A_{2}^{\frac{1}{2}} w\right\|_{C_{a, b}^{\prime}}\right)\|a\|_{C_{a, b}^{\prime}}\right\}[|d f(w)|+|d g(w)|]<+\infty
\end{aligned}
$$

Then for all $p \in[1,2]$ and all real $q_{j}$ with $\left|q_{j}\right| \geq\left|q_{0}\right|, j=1,2$,

$$
\begin{gathered}
T_{\vec{q}}^{(p)}\left((F * G)_{\vec{q}}\right)\left(y_{1}, y_{2}\right) \\
=\int_{C_{a, b}^{\prime}[0, T]} \int_{C_{a, b}^{\prime}[0, T]} \exp \left\{\frac{i}{\sqrt{2}}\left(A_{1}^{\frac{1}{2}}\left(w_{1}+w_{2}\right), y_{1}\right)^{\sim}+\frac{i}{\sqrt{2}}\left(A_{2}^{\frac{1}{2}}\left(w_{1}+w_{2}\right), y_{2}\right)^{\sim}\right. \\
-\frac{i}{2 q_{1}}\left[\left\|A_{1}^{\frac{1}{2}} w_{1}\right\|_{C_{a, b}^{\prime}}^{2}+\left\|A_{1}^{\frac{1}{2}} w_{2}\right\|_{C_{a, b}^{\prime}}^{2}\right] \\
\left.\quad-\frac{i}{2 q_{2}}\left[\left\|A_{2}^{\frac{1}{2}} w_{1}\right\|_{C_{a, b}^{\prime}}^{2}+\left\|A_{2}^{\frac{1}{2}} w_{2}\right\|_{C_{a, b}^{\prime}}^{2}\right]\right\} d f_{2 \vec{q}}^{\vec{A}, 2 a}\left(w_{1}\right) d g\left(w_{2}\right) \\
=T_{2 \vec{q}}^{(p)}\left(T_{2 \vec{q}}^{(p)}(F)\right)\left(\frac{y_{1}}{\sqrt{2}}, \frac{y_{2}}{\sqrt{2}}\right) \cdot T_{2 \vec{q}}^{(p)}\left(T_{2 \vec{q}}^{(p)}(G(-\cdot,-\cdot))(-\cdot,-\cdot)\right)\left(\frac{y_{1}}{\sqrt{2}}, \frac{y_{2}}{\sqrt{2}}\right) \\
\text { for s-a.e. }\left(y_{1}, y_{2}\right) \in C_{a, b}^{2}[0, T] .
\end{gathered}
$$


Theorem 4.9. Let $q_{0}$ be a nonzero real number and let $F$ and $G$ be elements of $\mathcal{F}_{A_{1}, A_{2}}$ whose associated measures $f$ and $g$ satisfy the condition

$$
\begin{aligned}
\int_{C_{a, b}^{\prime}[0, T]} \exp \left\{3 | 4 q _ { 0 } | ^ { - \frac { 1 } { 2 } } \left(\left\|A_{1}^{\frac{1}{2}} w\right\|_{C_{a, b}^{\prime}}\right.\right. \\
\left.\left.+\left\|A_{2}^{\frac{1}{2}} w\right\|_{C_{a, b}^{\prime}}\right)\|a\|_{C_{a, b}^{\prime}}\right\}[|d f(w)|+|d g(w)|]<+\infty
\end{aligned}
$$

Then for all $p \in[1,2]$ and all real $q_{j}$ with $\left|q_{j}\right| \geq\left|q_{0}\right|, j=1,2$,

$$
\begin{aligned}
& \int_{C_{a, b}^{2}[0, T]}^{a n f_{-\vec{q}}} T_{\vec{q}}^{(p)}\left((F * G)_{\vec{q}}\right)\left(y_{1}, y_{2}\right) d(\mu \times \mu)\left(y_{1}, y_{2}\right) \\
\equiv & \int_{C_{a, b}^{2}[0, T]}^{a n f_{-\vec{q}}} T_{2 \vec{q}}^{(p)}\left(T_{2 \vec{q}}^{(p)}(F)\right)\left(\frac{y_{1}}{\sqrt{2}}, \frac{y_{2}}{\sqrt{2}}\right) \\
\cdot & T_{2 \vec{q}}^{(p)}\left(T_{2 \vec{q}}^{(p)}(G(-\cdot,-\cdot))(-\cdot,-\cdot)\right)\left(\frac{y_{1}}{\sqrt{2}}, \frac{y_{2}}{\sqrt{2}}\right) d(\mu \times \mu)\left(y_{1}, y_{2}\right) \\
= & \int_{C_{a, b}^{2}[0, T]}^{a n f_{\vec{q}}} T_{-2 \vec{q}}^{(p)}\left(T_{2 \vec{q}}^{(p)}(F)\right)\left(\frac{y_{1}}{\sqrt{2}}, \frac{y_{2}}{\sqrt{2}}\right) \\
\cdot & T_{-2 \vec{q}}^{(p)}\left(T_{2 \vec{q}}^{(p)}(G)\right)\left(-\frac{y_{1}}{\sqrt{2}},-\frac{y_{2}}{\sqrt{2}}\right) d(\mu \times \mu)\left(y_{1}, y_{2}\right)
\end{aligned}
$$

for s-a.e. $\left(y_{1}, y_{2}\right) \in C_{a, b}^{2}[0, T]$. Also, both of the expressions in (4.27) are given by the expression

$$
\begin{aligned}
\int_{C_{a, b}^{\prime}[0, T]} \int_{C_{a, b}^{\prime}[0, T]} \exp \left\{-\frac{i}{4 q_{1}}\left\|A_{1}^{\frac{1}{2}}\left(w_{1}-w_{2}\right)\right\|_{C_{a, b}^{\prime}}^{2}\right. \\
-\frac{i}{4 q_{2}}\left\|A_{2}^{\frac{1}{2}}\left(w_{1}-w_{2}\right)\right\|_{C_{a, b}^{\prime}}^{2}+i\left(\frac{-i}{2 q_{1}}\right)^{\frac{1}{2}}\left(A_{1}^{\frac{1}{2}}\left(w_{1}-w_{2}\right), a\right)_{C_{a, b}^{\prime}} \\
\left.+i\left(\frac{-i}{2 q_{2}}\right)^{\frac{1}{2}}\left(A_{2}^{\frac{1}{2}}\left(w_{1}-w_{2}\right), a\right)_{C_{a, b}^{\prime}}\right\} d f_{2 \vec{q}}^{\vec{A}, 2 a}\left(w_{1}\right) d g_{-2 \vec{q}}^{\vec{A}, 2 a}\left(w_{2}\right) .
\end{aligned}
$$

\section{Example}

In this section we apply the results obtained in Section 4 to a specific linear operator $A$ on $C_{a, b}^{\prime}[0, T]$.

Let $S: C_{a, b}^{\prime}[0, T] \longrightarrow C_{a, b}^{\prime}[0, T]$ be the linear operator defined by

$$
S w(t)=\int_{0}^{t} w(s) d b(s) .
$$


Then, we see that the adjoint operator $S^{*}$ of $S$ is given by

$$
S^{*} w(t)=w(T) b(t)-\int_{0}^{t} w(s) d b(s)=\int_{0}^{t}[w(T)-w(s)] d b(s),
$$

and the linear operator $B=S^{*} S$ is given by

$$
B w(t)=\int_{0}^{T} \min \{b(s), b(t)\} w(s) d b(s) .
$$

Furthermore, we see that $B$ is a self-adjoint operator on $C_{a, b}^{\prime}[0, T]$ and that

$$
\left(w_{1}, B w_{2}\right)_{C_{a, b}^{\prime}}=\left(S w_{1}, S w_{2}\right)_{C_{a, b}^{\prime}}=\int_{0}^{T} w_{1}(s) w_{2}(s) d b(s)
$$

for all $w_{1}, w_{2} \in C_{a, b}^{\prime}[0, T]$. Hence $B$ is a positive definite operator, that is, $(w, B w)_{C_{a, b}^{\prime}} \geq 0$ for all $w \in C_{a, b}^{\prime}[0, T]$.

One can show that the orthonormal eigenfunctions $\left\{e_{m}\right\}$ of $B$ are given by

$$
e_{m}(t)=\frac{\sqrt{2 b(T)}}{\left(m-\frac{1}{2}\right) \pi} \sin \left(\frac{\left(m-\frac{1}{2}\right) \pi}{b(T)} b(t)\right)
$$

with corresponding eigenvalues $\beta_{m}$ given by

$$
\beta_{m}=\left(\frac{b(T)}{\left(m-\frac{1}{2}\right) \pi}\right)^{2} .
$$

Furthermore, it can be shown that $\left\{e_{m}\right\}$ is a basis of $C_{a, b}^{\prime}[0, T]$ and that $B$ is a trace class operator and so $S$ is a Hilbert-Schmidt operator on $C_{a, b}^{\prime}[0, T]$.

Define a self-adjoint operator on $C_{a, b}^{\prime}[0, T]$ by

$$
A w=\sum_{m=1}^{\infty} \gamma_{m}\left(w, e_{m}\right)_{C_{a, b}^{\prime}} e_{m}
$$

where

$$
\gamma_{m}= \begin{cases}\beta_{m}, & m: \text { even } \\ -\beta_{m}, & m: \text { odd }\end{cases}
$$

Then

$$
\begin{aligned}
& A w=\sum_{m=1}^{\infty}(-1)^{m} \beta_{m}\left(w, e_{m}\right)_{C_{a, b}^{\prime}} e_{m}, \\
& A_{+}^{\frac{1}{2}} w=\sum_{m: \text { even }} \sqrt{\beta_{m}}\left(w, e_{m}\right)_{C_{a, b}^{\prime}} e_{m},
\end{aligned}
$$

and

$$
A_{-}^{\frac{1}{2}} w=\sum_{m: \text { odd }} \sqrt{\beta_{m}}\left(w, e_{m}\right)_{C_{a, b}^{\prime}} e_{m}
$$


In this case, we see that $A_{+}$is the positive part of $A$ and $A_{-}$is the negative part of $A$. One can show that $A_{+}^{\frac{1}{2}}$ and $A_{-}^{\frac{1}{2}}$ are trace class operators with $\operatorname{Tr} A_{+}^{\frac{1}{2}}=\frac{b^{2}(T)}{8}$ and $\operatorname{Tr} A_{-}^{\frac{1}{2}}=\frac{3 b^{2}(T)}{8}$.

Let $F \in \mathcal{F}_{A_{+}, A_{-}}$. Then

$$
F(x)=\int_{C_{a, b}^{\prime}[0, T]} \exp \left\{i\left(A_{+}^{\frac{1}{2}} w, x\right)^{\sim}+i\left(A_{-}^{\frac{1}{2}} w, x\right)^{\sim}\right\} d f(w)
$$

for s-a.e. $\left(y_{1}, y_{2}\right) \in C_{a, b}^{2}[0, T]$. Suppose that the associated measure $f$ of $F$ satisfies the condition (4.9) with $A_{1}$ and $A_{2}$ replaced with $A_{+}$and $A_{-}$, respectively. Then for all $\vec{q}=(q,-q)$ with $q \in \mathbb{R}-\{0\}$ and $|q| \geq\left|q_{0}\right|$, using the equations (4.10) and (5.1)-(5.5), we have

$$
\begin{aligned}
T_{\vec{q}}^{(p)}(F)\left(y_{1}, y_{2}\right)= & \int_{C_{a, b}^{\prime}[0, T]} \exp \left\{i\left(A_{+}^{\frac{1}{2}} w, y_{1}\right)^{\sim}+i\left(A_{-}^{\frac{1}{2}} w, y_{2}\right)^{\sim}\right. \\
& -\frac{i}{2 q} \sum_{m=1}^{\infty}(-1)^{m}\left(\frac{b(T)}{\left(m-\frac{1}{2}\right) \pi}\right)^{2}\left(w, e_{m}\right)_{C_{a, b}^{\prime}}^{2} \\
& +i\left(\frac{i}{q}\right)^{\frac{1}{2}} \sum_{m: \text { even }} \frac{b(T)}{\left(m-\frac{1}{2}\right) \pi}\left(w, e_{m}\right)_{C_{a, b}^{\prime}}\left(a, e_{m}\right)_{C_{a, b}^{\prime}} \\
& \left.+i\left(\frac{-i}{q}\right)^{\frac{1}{2}} \sum_{m: \text { odd }} \frac{b(T)}{\left(m-\frac{1}{2}\right) \pi}\left(w, e_{m}\right)_{C_{a, b}^{\prime}}\left(a, e_{m}\right)_{C_{a, b}^{\prime}}\right\} d f(w)
\end{aligned}
$$

for s-a.e. $y \in C_{a, b}^{2}[0, T]$.

Also, for all $\vec{q}=(q,-q)$ with $q \in \mathbb{R}-\{0\}$ and $|q| \geq\left|q_{0}\right|$, using the equations (4.19), (5.1)-(5.5), we have

$$
\begin{aligned}
& =\int_{C_{a, b}^{\prime}[0, T]} \int_{C_{a, b}^{\prime}[0, T]} \exp \left\{\frac{i}{\sqrt{2}}\left(A_{+}^{\frac{1}{2}}\left(w_{1}+w_{2}\right), y_{1}\right)^{\sim}+\frac{i}{\sqrt{2}}\left(y_{-}^{\frac{1}{2}}\left(w_{1}+w_{2}\right), y_{1}\right)^{\sim}\right. \\
& \quad-\frac{i}{4 q} \sum_{m=1}^{\infty}(-1)^{m}\left(\frac{b(t)}{\left(m-\frac{1}{2}\right)}\right)^{2}\left(w_{1}-w_{2}, e_{m}\right)_{C_{a, b}^{\prime}}^{2} \\
& \quad+i\left(\frac{i}{2 q}\right)^{\frac{1}{2}} \sum_{m: \text { even }} \frac{b(T)}{\left(m-\frac{1}{2}\right) \pi}\left(w_{1}-w_{2}, e_{m}\right)_{C_{a, b}^{\prime}}\left(a, e_{m}\right)_{C_{a, b}^{\prime}} \\
& \left.\quad+i\left(\frac{-i}{2 q}\right)^{\frac{1}{2}} \sum_{m \text { odd }} \frac{b(T)}{\left(m-\frac{1}{2}\right) \pi}\left(w_{1}-w_{2}, e_{m}\right)_{C_{a, b}^{\prime}}\left(a, e_{m}\right)_{C_{a, b}^{\prime}}\right\} d f\left(w_{1}\right) d g\left(w_{2}\right)
\end{aligned}
$$

for s-a.e. $y \in C_{a, b}^{2}[0, T]$.

\section{References}

[1] J. M. Ahn, $L_{1}$ analytic Fourier-Feynman transform on the Fresnel class of abstract Wiener space, Bull. Korean Math. Soc. 35 (1998), no. 1, 99-117. 
[2] M. D. Brue, A functional transform for Feynman integrals similar to the Fourier transform, Thesis, Univ. of Minnesota, Minneapolis, 1972.

[3] R. H. Cameron and D. A. Storvick, An $L_{2}$ analytic Fourier-Feynman transform, Michigan Math. J. 23 (1976), no. 1, 1-30.

[4] S. J. Chang and J. G. Choi, Multiple $L_{p}$ analytic generalized Fourier-Feynman transform on the Banach algebra, Commun. Korean Math. Soc. 19 (2004), no. 1, 93-111.

[5] S. J. Chang, J. G. Choi, and D. Skoug, Integration by parts formulas involving generalized Fourier-Feynman transforms on function space, Trans. Amer. Math. Soc. 355 (2003), no. 7, 2925-2948.

[6] S. J. Chang and D. M. Chung, Conditional function space integrals with applications, Rocky Mountain J. Math. 26 (1996), no. 1, 37-62.

[7] K. S. Chang, B. S. Kim, and I. Yoo, Analytic Fourier-Feynman transform and convolution of functionals on abstract Wiener space, Rocky Mountain J. Math. 30 (2000), no. 3, 823-842.

[8] S. J. Chang and D. Skoug, Generalized Fourier-Feynman transforms and a first variation on function space, Integral Transforms Spec. Funct. 14 (2003), no. 5, 375-393.

[9] S. J. Chang and I. Y. Lee, Multiple $L_{p}$ analytic generalized Fourier-Feynman transform on a Fresnel type class, J. Chungcheong Math. Soc. 19 (2006), 79-99.

[10] _ Generalized Fourier-Feynman transforms and conditional generalized FourierFeynman transforms on a Fresnel type class, submitted for publication.

[11] K. S. Chang, T. S. Song, and I. Yoo, Analytic Fourier-Feynman transform and first variation on abstract Wiener space, J. Korean Math. Soc. 38 (2001), no. 2, 485-501.

[12] D. M. Chung, Scale-invariant measurability in abstract Wiener space, Pacific J. Math. 130 (1987), no. 1, 27-40.

[13] T. Huffman, C. Park, and D. Skoug, Analytic Fourier-Feynman transforms and convolution, Trans. Amer. Math. Soc. 347 (1995), no. 2, 661-673.

[14] Convolutions and Fourier-Feynman transforms of functionals involving multiple integrals, Michigan Math. J. 43 (1996), no. 2, 247-261.

[15] _ Convolution and Fourier-Feynman transforms, Rocky Mountain J. Math. 27 (1997), no. 3, 827-841.

[16] G. W. Johnson and D. L. Skoug, An $L_{p}$ analytic Fourier-Feynman transform, Michigan Math. J. 26 (1979), no. 1, 103-127.

[17] _ Scale-invariant measurability in Wiener space, Pacific J. Math. 83 (1979), no. $1,157-176$.

[18] G. Kallianpur and C. Bromley, Generalized Feynman integrals using analytic continuation in several complex variables, in Stochastic analysis and applications, 217-267, Adv. Probab. Related Topics, 7, Dekker, New York, 1984.

[19] H.-H. Kuo, Gaussian Measures in Banach Spaces, Lecture Notes in Math. 463, SpringerVerlag, Berlin, 1975

[20] J. Yeh, Convolution in Fourier-Wiener transform, Pacific J. Math. 15 (1965), 731-738.

[21] _ Stochastic Processes and the Wiener Integral, Marcel Dekker, Inc., New York, 1973.

[22] I. Yoo, Convolution and the Fourier-Wiener transform on abstract Wiener space, Rocky Mountain J. Math. 25 (1995), no. 4, 1577-1587.

Seung Jun Chang

Department of Mathematics

DANKOOK UNIVERSITY

Cheonan 330-714, Korea

E-mail address: sejchang@dankook.ac.kr 
IL YONG LEE

DePARTMENT OF MATHEMAtics

DANKOOK UNIVERSITY

Cheonan 330-714, Korea

E-mail address: iylee@dankook.ac.kr 